\title{
Parametric inference for multiple repairable systems under dependent competing risks
}

\author{
Anupap Somboonsavatdee ${ }^{\mathrm{a}}$ and Ananda Sen ${ }^{\mathrm{b} * \uparrow}$
}

The focus of this article is on the analysis of repairable systems that are subject to multiple sources of recurrence. The event of interest at the system level is assumed to be caused by the earliest occurrence of a source, thereby conforming to a series system competing risks framework. Parametric inference is carried out under the power law process model that has found significant attention in industrial applications. Dependence among the cause-specific recurrent processes is induced via a shared frailty structure. The theoretical inference results are implemented to a warranty database for a fleet of automobiles, for which the warranty repair is triggered by the failure of one of many components. Extensive finite-sample simulation is carried out to supplement the asymptotic findings. Copyright $@ 2014$ John Wiley \& Sons, Ltd.

Keywords: dependent failure modes; shared frailty; power law process; recurrent event; series system

\section{Introduction}

Data pertaining to failure history of a repairable system has long been a focus of interest to statisticians and reliability engineers. Situations where one studies repeated occurrences of an event of interest frequently arise in manufacturing, software development, medical applications, social sciences and risk analysis. In a medical application, 'failures' are often translated into times until the occurrence of a recurrent event (e.g., infection) in individuals. Clinical experiments typically consist of a fairly large number of individuals observed over a relatively short period. This is also common with databases of manufactured products generating warranty claims. By contrast, most of the field and bench test data for demonstrating product reliability consist of a very small number of prototypes put under test for a fairly long time. Irrespective of the area of application, interest often lies on the inference around the mean or rate function of the underlying process of recurrence, vis $\grave{a}$ vis their association with important prognostic factors. Statistical analysis is often carried out by assuming the recurrence history to follow a nonhomogeneous Poisson process, although this assumption can be relaxed.

Recently, there has been some interest in investigating repairable systems whose failures are subject to multiple failure modes, more widely known as competing risks. Under competing risks, the occurrence of a system failure is caused by the earliest onset of the component failures (a series system). The general topic of competing risks is well-studied in the context of non-repairable systems. The excellent books by Crowder [1] and Pintilie [2] motivate the need for accounting for competing risks in reliability and survival applications through several examples in industrial statistics and health sciences. In contrast with the investigations in the area of non-repairable systems, relatively fewer investigations have been carried out for analyzing failures of systems with multiple sources of recurrence. Yet, there are multiple examples available in the literature that fall under this framework. Majumdar [3] documents recurrent failure times of a vertical boring machine spanning a total of 18285 hours along with the indices of the components that are responsible for the failure. Langseth and Lindqvist [4] report cumulative service times of a component spanning over 1600 time units, marking each failure with the specific failure causing mode. The causes are categorized into two broad groups with several subcauses specified under each. Lawless et al. [5] analyze repeated shunt failures in infants diagnosed with hydrocephalus where the failures are known to occur due to a variety of causes. There are analogous examples in health sciences. Dauxois and Sencey [6] analyze occurrence rate of different types of recurrent infections for patients that are admitted to the French intensive care

\footnotetext{
${ }^{a}$ Department of Statistics, Faculty of Commerce and Accountancy, Chulalongkorn University, Bangkok, Thailand

${ }^{b}$ Department of Biostatistics, University of Michigan, Ann Arbor, MI, USA

*Correspondence to: Ananda Sen, Department of Biostatistics, University of Michigan, Ann Arbor, Michigan, USA.

${ }^{\dagger}$ E-mail: anandas@umich.edu
} 
unit followed over 4 years. All of these examples clearly demonstrate a need for a systematic development of methodologies to analyze recurrent failures under competing risks, the topic of our article.

Our focus in this article is fully parametric. The motivating premise is that of an industrial setup where the primary purpose of the inference is reliability planning, tracking, and prediction. This is common in defense industry where systems and subsystems undergo developmental testing programs with the goal of demonstrating a pre-specified growth in reliability. Further, in such situations, either there is limited data on covariates or a covariate adjusted analysis is not of primary importance. In order to integrate failure data from the early and late phase of the developmental program that can be used for projection and planning purposes, a parametric growth curve formulation is warranted.

In this paper, we discuss a specific parametric model known as a power law process (PLP) that has found considerable attention in the repairable systems literature [7,8]. The model, comprehensively studied first by Crow [9], has been used in various application areas including, most notably, the defense industry. Statistical inference of PLP model is investigated in the present article under competing risks.

The rest of the article is organized as follows. Section 2 introduces the competing risks framework for repairable systems. Section 3 formulates the setup for analyzing repairable systems subject to an arbitrary number of failure modes with inter-related failure processes. The inference results for PLP under dependent competing risks are developed in Section 4. Section 5 details findings from extensive finite-sample simulation that supplements the theoretical results in the previous section. Section 6 implements the inference results to a warranty database created for a fleet of automobiles for which the failure at the car level is triggered by the earliest failure of any one of its components. The article is concluded in Section 7 with some general remarks.

\section{Competing risks framework for repairable systems}

In order to understand the competing risks framework for analyzing repairable systems, consider first a single system with successive failures at calendar time $0<t_{1}<t_{2}<\ldots$. Suppose failures arise from an underlying competing risks structure that is equivalent to assuming the system failure to be caused by the earliest occurrence of one of $K$ mutually exclusive failure modes. At the $i$-th failure time $t_{i}$, one thus also observes the failure causing mode $\delta\left(t_{i}\right)$. Let $\{N(t), t \geqslant 0\}$ denote the cumulative failure counter at the system level. If $N_{k}(t)$ denotes the counting process corresponding to the $k$-th mode, then it easily follows that $N(t)=\sum_{=1}^{K} N_{k}(t)$. The mode specific intensity function is defined as

$$
\lambda_{k}(t)=\lim _{\Delta \rightarrow 0} \operatorname{Pr}[\delta(t)=k, N(t+\Delta)-N(t)=1 \mid N(s), 0 \leqslant s \leqslant t] / \Delta
$$

It is easy to see that the time and the mode of failure are stochastically independent if and only if $\lambda_{k}(t)$ 's are proportional to each other [10]. This is a simple extension of a corresponding result from the competing risks literature in failure time modeling of non-repairable systems [11].

Generally, there are two different approaches that have been pursued in analyzing recurrent failures. One approach is to base the inference on models of gap times between successive failures. Such modeling can be entertained also in presence of competing risks $[5,12]$. The capability of the gap-time models to directly incorporate any effect of design or configuration changes is clearly quite attractive. However, gap-time formulation is often confronted with modeling challenges such as length biasing and informative censoring [13]. The alternative approach to directly investigate the associated counting process is attractive because it avoids such challenges through well-defined structures (e.g., Poisson process). Clearly there is a one-to-one correspondence between the two approaches as models on gap-times induces a model on the corresponding counting processes and vice versa. The choice of framework, however, is often dictated by the physical motivation that underscores the process of recurrence.

The modeling strategy we adopt in this paper is the latter of the two described in the paragraph earlier. We study systems with a series connection of $K$ components (or failure modes) whose failures are governed by separate PLPs with the respective intensities sharing a latent frailty variable. In the next section, we describe the Power law process model under this shared frailty structure that we study in the context of competing risks.

\section{Power law model under shared frailty}

\subsection{Power law process model}

Power law process has been popular primarily because of its attractive physical interpretation in the way of depicting the failure history of a repairable system. PLP describes the repeated failure history by assuming an nonhomogeneous Poisson 
process for the underlying counting process $N(t), t \geqslant 0$, with intensity function

$$
\lambda(t)=\mu \beta t^{\beta-1}, t \geqslant 0, \mu>0, \beta>0,
$$

matching the hazard rate of a Weibull distribution. The linear relation of the intensity in (1) with time in log-log scale conforms to empirical observations in hardware failure processes encountered in manufacturing industry [14]. The PLP is a flexible model in that it can represent a decay $(\beta>1)$, a growth $(\beta<1)$, or a flat pattern $(\beta=1)$ in reliability. One of the features of PLP is the surprising elegance of the statistical inference [9]. It has been extensively used in analyzing repairable systems data by reliability practitioners in manufacturing and defense industry where data is limited, and there is a strong desire to construct a reliability growth curve that can be used for tracking, planning, and projection. Often, in this context, the tested devices or systems are complex, typically comprising of multiple inter-related components. Extensive investigation regarding PLP have appeared in the literature in the 70s and the 80s. The book by Rigdon and Basu [8] provides a comprehensive account of the research related to PLP.

As indicated earlier, in our competing risks setup, the underlying failure process for the failure modes are generated by PLPs. No restriction is imposed on the failure pattern of the modes so that each can exhibit either a decay, growth, or flat pattern in reliability. Under a series structure, the system level failure process conforms to a PLP if and only if the growth parameters $(\beta)$ of all components are equal. In the general case, the system level intensity encompasses a range of possible features including a bathtub shaped structure.

\subsection{Shared frailty formulation}

In time to event studies, the idea of frailty is used to model unobserved heterogeneity among subjects or units. In that sense, frailty models are special classes of random effects models. The term 'frailty', coined in [15] in estimating mortality rates, points to the inherent heterogeneity in the population where a subgroup is perhaps more 'frail' and hence is more prone to early failures. In a typical application, frailty appears as a multiplicative random effect to a hazard rate. Conditionally, given a random variable $Z$, one assumes the hazard function to be of the form

$$
h(t \mid Z)=Z h_{0}(t)
$$

where $h_{0}(t)$ satisfies the properties of a hazard function. The associated conditional survival function is $S(t \mid Z)=$ $\exp \left[-Z \int_{0}^{t} h_{0}(y) d y\right]$. Frailty thus gives rise to a mixture distribution. The observed marginal survivor function is obtained by integrating out $Z$, i.e.

$$
S(t)=E_{Z}[S(t \mid Z)]=\mathcal{L}\left(\int_{0}^{t} h_{0}(y) d y\right)
$$

where $\mathcal{L}$ is the Laplace transform of the frailty distribution. The marginal inference is greatly facilitated when the distribution of the frailty yields a tractable Laplace transform. Some common examples of frailty distribution include exponential, gamma, Weibull, Gompertz, inverse Gaussian, and a positive stable family of distributions with Laplace transform $\mathcal{L}(s)=\exp \left(-s^{\alpha}\right), 0 \leqslant \alpha \leqslant 2$. An excellent and comprehensive treatment on frailty and its theoretical nuances has been carried out in [16].

Shared frailty refers to the framework where individuals or units belonging to a cluster share the same value of frailty. Physically, it may represent an effect of a common environmental stress the cluster is subjected to. In the recurrent events context, such clustering appears naturally across the repeated events of a system. Thus, for the $i$-th system, the counting process associated with the recurrent event history can be modeled with an intensity function

$$
\lambda(i, t)=Z_{i} \mu(t)
$$

conditionally given $Z_{i}$. For $m$ systems under observation, $Z_{i}, i=1, \ldots, m$ are usually modeled as independent and identically distributed copies of a random variable. Further, the $Z_{i}$ 's are assumed to be stochastically independent of the failure process governed by the intensity function $\mu(t)$. The common value of $Z_{i}$ creates dependence within observations in the $i$-th cluster. The model in (2) is non-identifiable in a nonparametric sense. A fixed parametric structure of $\mu(t)$ and a specified parametric distribution for $Z_{i}$ makes (2) identifiable. Interestingly, a proportional regression form of $\mu(t)=\mu_{0}(t) \exp \left(\gamma^{\prime} x\right)$ makes the model identifiable even without having to parameterize $Z$ [17]. 


\subsection{Competing risks power law process model under shared frailty}

The PLP under shared frailty conforms to a $K$-component series system where the failures of the $k$-th component is governed by a PLP $N_{k}(t)$ with intensity function

$$
\lambda_{k}(t)=Z \mu_{k} \beta_{k} t^{\beta_{k}-1}, k=1, \ldots, K
$$

conditionally given a latent random variable $Z$. Although given the frailty $Z$, the $N_{k}$ 's are assumed to be independent, the dependence among $N_{k}$ 's follows in an unconditional sense, upon integrating over the distribution of the shared frailty $Z$. The marginal mean and variance of the component processes are given by

$$
\mathrm{E}\left(N_{k}(t)\right)=\mu_{k} t^{\beta_{k}} E(Z), \quad \operatorname{Var}\left(N_{k}(t)\right)=\mu_{k} t^{\beta_{k}}\left[E(Z)+\mu_{k} t^{\beta_{k}} \operatorname{Var}(Z)\right] .
$$

Moreover, for a given $t$, the component specific counting processes $N_{k}(t), k=1,2, \ldots, K$ are correlated with each other. Specifically, for $k \neq k^{\prime}$,

$$
\begin{aligned}
\operatorname{Cov}\left(N_{k}(t), N_{k^{\prime}}(t)\right) & =\mathrm{E}\left[\operatorname{Cov}\left(N_{k}(t), N_{k^{\prime}}(t) \mid Z\right)\right]+\operatorname{Cov}\left[\mathrm{E}\left(N_{k}(t) \mid Z\right), \mathrm{E}\left(N_{k^{\prime}}(t) \mid Z\right)\right] \\
& =0+\left(\mu_{k} t^{\beta_{k}}\right)\left(\mu_{k^{\prime}} t_{k}^{\beta_{k}^{\prime}}\right) \operatorname{Var}(Z) \\
& =\mu_{k} \mu_{k^{\prime}} t^{\beta_{k}+\beta_{k^{\prime}}} \operatorname{Var}(Z) .
\end{aligned}
$$

An immediate consequence of (5) is that the component processes are positively correlated. In the sequel, we take $Z$ to be a gamma random variable. The choice of gamma is dictated largely by mathematical tractability. With gamma frailty, the marginal distribution of $N_{k}(t)$ becomes negative binomial, which is often used as a candidate for the distribution of an overdispersed Poisson random variable. A quick inspection of (3) reveals that a full specification of gamma will lead to scale non-identifiability. We take the mean of $Z$ to be equal to one to circumvent this problem. Specifically, the probability density function (pdf) of $Z$ will assume the form

$$
g(z)=\frac{1}{\Gamma\left(\eta^{-1}\right) \eta^{\eta^{-1}}} z^{\eta^{-1}-1} \exp (-z / \eta), \quad \eta>0
$$

which is the pdf of a gamma random variable with scale $=\eta$ and shape $=\eta^{-1}$. The parameter $\eta=\operatorname{Var}(Z)$ represents the variability between systems. As $\eta \longrightarrow 0$ so that $Z$ tends to a degenerate random variable at 1 , the component processes approach being independent. On the other hand, as $\eta$ grows large, so does the correlation between the component processes.

\section{Likelihood based inference}

In our framework, we shall assume the existence of $m$ identical systems experiencing repeated failures that result from the failure of any of the $K$ components connected in series. Let $t_{i j}$ denote the $j$-th cumulative failure time of the $i$-th system, $i=1,2, \ldots, m$. Along with the failure times, we also have information on $\delta_{i j}$, index of the mode responsible for the corresponding failure. The $i$-th system is observed until a censoring time $\tau_{i}$, which is assumed to be noninformative of the failure process. Let $n_{i}$ be the observed number of failures for the $i$-th system, with $n=\sum_{i=1}^{m} n_{i}$ denoting the total number of observed system failures. Then, conditionally given $Z_{i}$, using standard properties of a Poisson process, the likelihood contribution from the $i$-th system with (3) governing the underlying failure process is

$$
\begin{aligned}
L_{i}\left(\mu_{1}, \mu_{2}, \ldots, \mu_{K}, \beta_{1}, \beta_{2}, \ldots, \beta_{K} \mid Z_{i}\right)= & \prod_{j=1}^{n_{i}} \prod_{k=1}^{K}\left[Z_{i} \mu_{k} \beta_{k} t_{i j}^{\beta_{k}-1}\right]^{I\left(\delta_{i j}=k\right)} \\
& \times \exp \left[-Z_{i} \sum_{k=1}^{K} \mu_{k} \tau_{i}^{\beta_{k}}\right],
\end{aligned}
$$

$I(\cdot)$ denoting the indicator function. We shall provide the details for the case of single censoring, namely, $\tau_{i}=\tau$ for simplicity of exposition. Integrating (7) over the distribution of $Z_{i}$, the unconditional likelihood contribution from the $i$-th system is 


$$
L_{i}\left(\mu_{1}, \ldots, \mu_{K} ; \beta_{1}, \ldots, \beta_{K}\right)=\prod_{k=1}^{K}\left\{\left(\mu_{k} \beta_{k}\right)^{n_{i k}} \prod_{j=1}^{n_{i}}\left(t_{i j}^{\beta_{k}-1}\right)^{I\left(\delta_{i j}=k\right)}\right\} \times \mathrm{E}_{Z}\left[Z^{n_{i}} \exp \left(-Z \sum_{k=1}^{K} \mu_{k} \tau^{\beta_{k}}\right)\right],
$$

where $n_{i k}$ denotes the number of $k$-component failures observed for the $i$-th system, and $\mathrm{E}_{Z}(\cdot)$ refers to the expectation with respect to the distribution of $Z$. Combined likelihood function based on all $m$ systems is calculated as $L=\prod_{i=1}^{m} L_{i}$, which upon maximizing with respect to $\mu_{k}, \beta_{k}$, yield the respective maximum likelihood estimators (MLE). We assume $Z_{1} \ldots, Z_{m}$ to be a random sample from the gamma pdf defined by (6). With such a choice, the unconditional MLEs of $\mu_{k}, \beta_{k}$ are in the remarkably simple form:

$$
\hat{\beta}_{k}=\frac{\sum_{i=1}^{m} n_{i k}}{\sum_{i=1}^{m} \sum_{j=1}^{n_{i}} I\left(\delta_{i j}=k\right) \log \left(\tau / t_{i j}\right)}, \quad \hat{\mu}_{k}=\frac{\sum_{i=1}^{m} n_{i k}}{m \tau^{\hat{\beta}_{k}}}, \quad k=1,2, \ldots, K .
$$

Note that the estimators in (9) are identical to the case when the component failure processes are independent. Further, it is evident that for $\hat{\mu}_{k}, \hat{\beta}_{k}$ to be defined, at least one failure needs to be caused by the $k$-th mode. The MLE $\hat{\eta}$ results by solving $\partial \log L / \partial \eta=0$, which reduces to the equation

$$
\begin{aligned}
0= & -\sum_{i=1}^{m} \psi\left(n_{i}+\eta^{-1}\right)+m \psi\left(\eta^{-1}\right)+m \log \eta-m+\sum_{i=1}^{m}\left(n_{i}+\eta^{-1}\right) /\left(\sum_{k=1}^{K} \mu_{k} \tau^{\beta_{k}}+\eta^{-1}\right) \\
& +m \log \left(\sum_{k=1}^{K} \mu_{k} \tau^{\beta_{k}}+\eta^{-1}\right),
\end{aligned}
$$

with $\psi$ denoting the digamma function. Plugging in the expressions from (9), the score equation (10) is tantamount to solving $f(\eta)=0$, where

$$
f(\eta)=-\sum_{i=1}^{m} \psi\left(n_{i}+\eta^{-1}\right)+m \psi\left(\eta^{-1}\right)+m \log \left(1+\frac{m \eta}{n}\right) .
$$

Finite-sample inference for the maximum likelihood estimators does not yield any tractable distributional results. We provide in the succeeding text the large-sample distribution of $\left(\hat{\mu}_{k}, \hat{\beta}_{k}, \hat{\mu}_{k^{\prime}}, \hat{\beta}_{k^{\prime}}\right)$ for any pair $k, k^{\prime} \in\{1,2, \ldots, K\}, k \neq k^{\prime}$, proof of which is relegated to the Appendix. In the sequel, we shall reserve the square brackets to denote matrices.

\section{Theorem 1}

Let us define $Q_{1 m}=\sqrt{m}\left(\hat{\mu}_{k}-\mu_{k}\right), Q_{2 m}=\sqrt{m}\left(\hat{\beta}_{k}-\beta_{k}\right), Q_{3 m}=\sqrt{m}\left(\hat{\mu}_{k^{\prime}}-\mu_{k^{\prime}}\right), Q_{4 m}=\sqrt{m}\left(\hat{\beta}_{k^{\prime}}-\beta_{k^{\prime}}\right)$ and $Q_{m}=$ $\left(Q_{1 m}, Q_{2 m}, Q_{3 m}, Q_{4 m}\right)^{\prime}$. Then, $Q_{m} \stackrel{d}{\rightarrow} N(0, \Omega)$ where

$$
\Omega=\left[\begin{array}{ll}
\omega_{11} & \omega_{12} \\
\omega_{12}^{\prime} & \omega_{22}
\end{array}\right]
$$

with

$$
\begin{aligned}
& \omega_{11}=\frac{1}{\tau^{\beta_{k}}}\left[\begin{array}{cc}
\mu_{k}\left(\beta_{k} \log \tau\right)^{2}+\mu_{k}\left(1+\eta \mu_{k} \tau^{\beta_{k}}\right) & -\beta_{k}^{2} \log \tau \\
-\beta_{k}^{2} \log \tau & \beta_{k}^{2} / \mu_{k}
\end{array}\right], \\
& \omega_{12}=\left[\begin{array}{cc}
\mu_{k} \mu_{k^{\prime}} & 0 \\
0 & 0
\end{array}\right]
\end{aligned}
$$

and $\omega_{22}$ is identical to $\omega_{11}$ with $k$ replaced by $k^{\prime}$. 
Theorem 1 indicates some interesting features of the maximum likelihood estimators. The large-sample distribution of $\hat{\mu}_{k}$ involves $\eta$. The difference between the asymptotic variances in the cases of independence and frailty-induced dependence increases linearly with $\eta$, and so does the large-sample covariance between $\hat{\mu}_{k}$ and $\hat{\mu}_{k^{\prime}}$. On the other hand, we note that the large-sample distribution of $\hat{\beta}_{k}$ is identical to that in the case where the components work independently of each other. Further, $\hat{\beta}_{k}$ is asymptotically independent of the ML estimators of the parameters specific to any other component. At first glance, one has the impression that the shared frailty does not have any effect on the asymptotic distribution of $\hat{\beta}_{k}$. In reality, however, the large-sample behavior of $\hat{\beta}_{k}$ is a consequence of imposing a unit mean condition for $Z$. We demonstrate in the Appendix the identity

$$
\sqrt{m}\left(\hat{\beta}_{k}-\beta_{k}\right)=\frac{-\hat{\beta}_{k}}{\sqrt{\sum_{i=1}^{m} n_{i k} / m}} v_{k m}
$$

where $v_{k m}$ is a function of $\hat{\beta}_{k}$ and $\sum_{i=1}^{m} n_{i k}$. Distribution of the negative binomial random variable $\sum_{i=1}^{m} n_{i k}$ depends on the parameters of $Z$, which in turn induces dependence of $\hat{\beta}_{k}$ on $Z$. As the number of systems grows large,

$$
\sum_{i=1}^{m} n_{i k} / m \stackrel{p}{\longrightarrow} \mu_{k} \tau^{\beta_{k}} E(Z) \quad \text { and } \quad \nu_{k m} \stackrel{\mathrm{d}}{\longrightarrow} \mathrm{N}(0,1)
$$

by the weak law of large numbers and the central limit theorem, respectively. When $E(Z)=1$, the large-sample variance for $\hat{\beta}_{k}$ matches that in the no-frailty case.

The parametric model based estimator of the mean number of system failures $\Lambda(t)$ is $\hat{\Lambda}(t)=\sum_{k=1}^{K} \hat{\mu}_{k} t_{\hat{t}_{k}}$, which, at the censoring time $\tau$, matches the nonparametric estimator $n / m$. A $100(1-\alpha) \%$ approximate pointwise confidence interval for $\Lambda(t)$ is given by

$$
\hat{\Lambda}(t) \pm z_{1-\alpha / 2} \sqrt{\hat{\Lambda}(t)(1+\eta \hat{\Lambda}(t))} .
$$

The standard error explicitly indicates the extra-Poisson variation induced by dependence.

\section{Remarks}

1 As is shown in the proof in the Appendix, we do not explicitly use the specific distributional form of frailty in order to derive the results in Theorem 1. The results are valid more generally for frailty distributions with unit mean. Quite interestingly, starting from (8) for a general frailty variable $Z$, one can derive the MLE of $\beta_{k}$ to be identical to that in (9). For arbitrary $Z$, the MLE of $\mu$ does not simplify to the expression in (9), however.

2 The contrast between the large-sample results in the single system and the multiple system case is worth a mention. Somboonsavatdee and Sen [10] have demonstrated some peculiarities of the inference of the ML estimators in the single-sample case where the large-sample results are applied as the number of repeated failures from the system grows large. In that case, the estimators of $\mu_{k}$ 's suffer from a pretty slow rate of convergence. Further, the asymptotic variance-covariance matrix turns out to be singular, highlighting the contrast with the multiple system case, which is devoid of such pathologies.

\subsection{Contrasting with the nonparametric approach}

Extensive research has been pursued in the area of recurrent events in the nonparametric setting ([18-20], among others). Recurrent events under competing risks have been studied recently by Dauxois and Sencey [6]. The nonparametric development focuses on the mean and rate functions and in the absence of any covariates is valid only for $t<\tau$, where $\tau$ is the largest censoring time observed. When all systems are observed until a single censoring time $\tau$, the inference is particularly simple. Specifically, the Nelson-Aalen estimators for the cause-specific cumulative intensity $\Lambda_{k}(t)$ and the overall cumulative intensity $\Lambda(t)=\sum_{k=1}^{K} \Lambda_{k}(t)$ are given by

$$
\hat{\Lambda}_{k}^{N A}(t)=\frac{\sum_{i=1}^{m} n_{i k}(t)}{m}, k=1, \ldots, K ; \quad \hat{\Lambda}^{N A}(t)=\frac{\sum_{i=1}^{m} \sum_{k=1}^{K} n_{i k}(t)}{m},
$$


where $n_{i k}(t)$ is the observed number of $k$-mode failures for the $i$-th system in time $[0, t]$. Note that the estimators in (12) are valid up to the censoring time $\tau$. As the number of systems grows large $(m \longrightarrow \infty)$, the centered and scaled process $m^{1 / 2}\left\{\hat{\Lambda}_{k}^{N A}(t)-\Lambda_{k}(t)\right\}$ converges to a Gaussian process with a mean zero and a covariance function $\xi_{k}(s, t)$ that is consistently estimated by

$$
\hat{\xi}_{k}(s, t)=m^{-1} \sum_{i=1}^{m}\left(n_{i k}(t)-\frac{\sum_{i=1}^{m} n_{i k}(t)}{m}\right)\left(n_{i k}(s)-\frac{\sum_{i=1}^{m} n_{i k}(s)}{m}\right), 0<s \leqslant t \leqslant \tau .
$$

An analogous result holds for the overall system where $m^{1 / 2}\left\{\hat{\Lambda}^{N A}(t)-\Lambda(t)\right\}$ converges to mean zero Gaussian process having a covariance function $\xi(s, t)$, which has a consistent estimator

$$
\hat{\xi}(s, t)=m^{-1} \sum_{i=1}^{m}\left(n_{i \cdot}(t)-\frac{\sum_{i=1}^{m} n_{i}(t)}{m}\right)\left(n_{i \cdot}(s)-\frac{\sum_{i=1}^{m} n_{i \cdot}(s)}{m}\right), 0<s \leqslant t \leqslant \tau,
$$

with $n_{i}(t)=\sum_{k=1}^{K} n_{i k}(t)$. The corresponding results for the multiple censoring case involve more complex expressions and are presented in [6]. In the data analysis section presented in Section 6, we contrast the parametric and the nonparametric estimators of the cumulative intensity function.

\section{Simulation study}

We carried out extensive simulation in order to investigate finite-sample properties of the MLEs, especially for a small number of systems. In order to keep the exposition simple, we kept our investigation confined to a two-mode system. Among the many parameter combinations tested, we report the findings for three sets of $\left(\mu_{1}, \beta_{1}, \mu_{2}, \beta_{2}\right)$, namely $(4,0.25$, $0.25,2),(1,0.75,0.3,1.25)$, and $(0.5,1.5,5,0.8)$. A single-censoring scheme was simulated with censoring time $\tau$ fixed at 20 . The three scenarios yield the mean number of failures to be $(8.5,100),(9.5,12.7),(44.7,55)$ by time $\tau$, respectively, for the two modes. Whereas the first of these corresponds to the situation with one dominating mode, the remaining two cases represent a two-mode system where the modes contribute similarly, with the two cases differing in terms of the propensity of failures. The number of systems $(\mathrm{m})$ tested ranged between 10 and 50, whereas the frailty variance $\eta$ was varied from a small value of 0.1 to a large value of 5 representing a low to a high degree of dependence.

Performance measures for the MLEs are calculated for the three sets of parameter values with $m=10,25,50$, and $\eta=0.1,1,5$, on the basis of 5000 replications of data generated from PLPs under competing risks with shared frailty. Specifically, the data for each replication with a given parametric configuration are obtained using the following steps:

Step 1: Generate a random observation $z_{i}$ from $\operatorname{Gamma}\left(\eta^{-1}, \eta\right)$ conforming to (6).

Step 2: Generate random observations $n_{i 1}, n_{i 2}$ from Poisson distributions with mean $=z_{i} \mu_{1} \tau^{\beta_{1}}, z_{i} \mu_{2} \tau^{\beta_{2}}$, respectively.

Step 3: Following the properties of PLP, generate the successive mode-1 failure times $t_{i 1}^{(1)}, t_{i 2}^{(1)}, \ldots, t_{i n_{i 1}}^{(1)}$ as $t_{i j}^{(1)}=\tau\left(u_{(j)}\right)^{1 / \beta_{1}}$, where $u_{(1)}, u_{(2)}, \ldots, u_{\left(n_{i 1}\right)}$ are order statistics based on a random sample of size $n_{i 1}$ from $\operatorname{Uniform}(0,1)$.

\begin{tabular}{|c|c|c|c|c|c|c|c|c|c|c|}
\hline No. of Systems & $\operatorname{Bias}\left(\hat{\mu}_{1}\right)$ & $\operatorname{FE}\left(\hat{\mu}_{1}\right)$ & $\mathrm{FE}_{\text {Ind }}\left(\hat{\mu}_{1}\right)$ & $\operatorname{Bias}\left(\hat{\beta}_{1}\right)$ & $\operatorname{FE}\left(\hat{\beta}_{1}\right)$ & $\operatorname{Bias}\left(\hat{\mu}_{2}\right)$ & $\operatorname{FE}\left(\hat{\mu}_{2}\right)$ & $\mathrm{FE}_{\text {Ind }}\left(\hat{\mu}_{2}\right)$ & $\operatorname{Bias}\left(\hat{\beta}_{2}\right)$ & $\operatorname{FE}\left(\hat{\beta}_{2}\right)$ \\
\hline (m) & \multicolumn{10}{|c|}{$\mu_{1}=4, \quad \beta_{1}=0.25, \quad \mu_{2}=0.25, \quad \beta_{2}=2$} \\
\hline 10 & -0.007 & 0.989 & 0.642 & 0.003 & 0.983 & 0.003 & 1.004 & 0.790 & 0.003 & 0.997 \\
\hline 25 & -0.013 & 0.953 & 0.618 & 0.001 & 0.950 & 0.001 & 1.021 & 0.803 & 0.001 & 1.009 \\
\hline 50 & 0.007 & 0.957 & 0.621 & 0.000 & 0.958 & 0.001 & 0.985 & 0.775 & 0.000 & 1.000 \\
\hline \multicolumn{11}{|c|}{$\mu_{1}=1, \quad \beta_{1}=0.75, \quad \mu_{2}=0.3, \quad \beta_{2}=1.25$} \\
\hline 10 & 0.005 & 0.986 & 0.853 & 0.007 & 0.919 & 0.007 & 1.000 & 0.923 & 0.010 & 0.958 \\
\hline 25 & 0.002 & 0.977 & 0.845 & 0.003 & 0.967 & 0.003 & 0.989 & 0.912 & 0.004 & 0.977 \\
\hline 50 & 0.000 & 0.995 & 0.860 & 0.002 & 0.983 & 0.002 & 0.981 & 0.905 & 0.002 & 1.013 \\
\hline \multicolumn{11}{|c|}{$\mu_{1}=0.5, \quad \beta_{1}=1.5, \quad \mu_{2}=5, \quad \beta_{2}=0.8$} \\
\hline 10 & 0.007 & 0.954 & 0.788 & 0.003 & 0.935 & -0.007 & 1.004 & 0.553 & 0.001 & 1.003 \\
\hline 25 & 0.004 & 0.960 & 0.793 & 0.001 & 0.985 & 0.006 & 1.005 & 0.554 & 0.001 & 0.995 \\
\hline 50 & 0.002 & 1.026 & 0.847 & 0.000 & 1.018 & 0.005 & 0.983 & 0.542 & 0.000 & 1.002 \\
\hline
\end{tabular}


Table II. Bias and finite-sample efficiencies of the maximum likelihood estimators for $\tau=20$ and $\eta=1$.

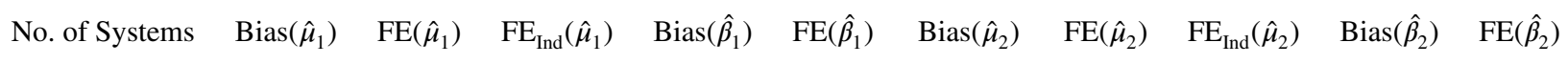

\begin{tabular}{|c|c|c|c|c|c|c|c|c|c|c|}
\hline (m) & \multicolumn{10}{|c|}{$\mu_{1}=4, \quad \beta_{1}=0.25, \quad \mu_{2}=0.25, \quad \beta_{2}=2$} \\
\hline 10 & -0.024 & 1.008 & 0.157 & 0.003 & 0.842 & 0.003 & 0.980 & 0.264 & 0.002 & 0.879 \\
\hline 25 & -0.014 & 0.988 & 0.154 & 0.001 & 0.889 & 0.001 & 0.988 & 0.266 & 0.001 & 0.956 \\
\hline \multirow[t]{2}{*}{50} & -0.007 & 0.984 & 0.153 & 0.001 & 0.937 & 0.001 & 0.981 & 0.264 & 0.001 & 1.000 \\
\hline & \multicolumn{10}{|c|}{$\mu_{1}=1, \quad \beta_{1}=0.75, \quad \mu_{2}=0.3, \quad \beta_{2}=1.25$} \\
\hline 10 & 0.012 & 0.983 & 0.383 & 0.008 & 0.857 & 0.007 & 0.985 & 0.534 & 0.012 & 0.879 \\
\hline 25 & 0.000 & 1.008 & 0.393 & 0.004 & 0.920 & 0.001 & 1.006 & 0.546 & 0.007 & 0.956 \\
\hline \multirow[t]{2}{*}{50} & 0.000 & 1.007 & 0.393 & 0.001 & 0.974 & 0.001 & 1.000 & 0.542 & 0.003 & 0.949 \\
\hline & \multicolumn{10}{|c|}{$\mu_{1}=0.5, \quad \beta_{1}=1.5, \quad \mu_{2}=5, \quad \beta_{2}=0.8$} \\
\hline 10 & 0.000 & 1.028 & 0.330 & 0.004 & 0.921 & -0.024 & 1.012 & 0.111 & 0.001 & 0.868 \\
\hline 25 & 0.000 & 1.027 & 0.330 & 0.000 & 0.956 & -0.043 & 1.050 & 0.115 & 0.000 & 0.957 \\
\hline 50 & 0.002 & 1.007 & 0.324 & 0.001 & 0.998 & 0.009 & 1.016 & 0.111 & 0.000 & 1.004 \\
\hline
\end{tabular}

Table III. Bias and finite-sample efficiencies of the maximum likelihood estimators for $\tau=20, \eta=5$.

\begin{tabular}{|c|c|c|c|c|c|c|c|c|c|c|}
\hline No. of Systems & $\operatorname{Bias}\left(\hat{\mu}_{1}\right)$ & $\operatorname{FE}\left(\hat{\mu}_{1}\right)$ & $\mathrm{FE}_{\text {Ind }}\left(\hat{\mu}_{1}\right)$ & $\operatorname{Bias}\left(\hat{\beta}_{1}\right)$ & $\operatorname{FE}\left(\hat{\beta}_{1}\right)$ & $\operatorname{Bias}\left(\hat{\mu}_{2}\right)$ & $\operatorname{FE}\left(\hat{\mu}_{2}\right)$ & $\mathrm{FE}_{\mathrm{Ind}}\left(\hat{\mu}_{2}\right)$ & $\operatorname{Bias}\left(\hat{\beta}_{2}\right)$ & $\operatorname{FE}\left(\hat{\beta}_{2}\right)$ \\
\hline (m) & \multicolumn{10}{|c|}{$\mu_{1}=4, \quad \beta_{1}=0.25, \quad \mu_{2}=0.25, \quad \beta_{2}=2$} \\
\hline 10 & -0.051 & 1.024 & 0.036 & 0.007 & 0.309 & 0.001 & 1.025 & 0.070 & 0.004 & 0.544 \\
\hline 25 & -0.035 & 1.022 & 0.036 & 0.001 & 0.757 & -0.001 & 1.036 & 0.071 & 0.002 & 0.770 \\
\hline \multirow[t]{2}{*}{50} & -0.032 & 0.985 & 0.035 & 0.001 & 0.892 & -0.001 & 0.979 & 0.067 & 0.001 & 0.863 \\
\hline & \multicolumn{10}{|c|}{$\mu_{1}=1, \quad \beta_{1}=0.75, \quad \mu_{2}=0.3, \quad \beta_{2}=1.25$} \\
\hline 10 & 0.005 & 0.994 & 0.113 & 0.020 & 0.252 & 0.009 & 1.005 & 0.192 & 0.021 & 0.192 \\
\hline 25 & 0.013 & 0.943 & 0.107 & 0.005 & 0.750 & 0.006 & 0.961 & 0.184 & 0.006 & 0.763 \\
\hline \multirow[t]{2}{*}{50} & 0.007 & 1.000 & 0.113 & 0.001 & 0.888 & 0.003 & 0.980 & 0.188 & 0.003 & 0.881 \\
\hline & \multicolumn{10}{|c|}{$\mu_{1}=0.5, \quad \beta_{1}=1.5, \quad \mu_{2}=5, \quad \beta_{2}=0.8$} \\
\hline 10 & 0.010 & 0.964 & 0.083 & 0.006 & 0.452 & 0.023 & 0.957 & 0.023 & 0.004 & 0.374 \\
\hline 25 & 0.000 & 1.036 & 0.090 & 0.002 & 0.751 & -0.005 & 1.047 & 0.025 & 0.000 & 0.808 \\
\hline 50 & -0.003 & 1.003 & 0.087 & 0.001 & 0.888 & -0.042 & 1.007 & 0.024 & 0.001 & 0.869 \\
\hline
\end{tabular}

Step 4: Similarly generate $n_{i 2}$ successive failure times corresponding to mode 2 .

Step 5: Repeat this for $i=1,2, \ldots, m$.

Tables I-III exhibit the bias and the finite-sample efficiencies (FE) of the MLEs. FE is defined as the ratio of the largesample (theoretical) variance obtained from Theorem 1, and the variance calculated from the simulated samples. For the $\mu$ parameters, a second efficiency measure $\left(\mathrm{FE}_{\mathrm{Ind}}\right)$ is calculated for comparative purposes, where the numerator of $\mathrm{FE}$ is replaced by the large-sample expression under the assumption of independence of the failure modes. This provides an assessment of misspecification arising from ignoring the underlying dependence between the failure modes. As is evident from Tables I to III, the bias in the ML estimates for both the scale and the shape parameters are quite small, even for small $m$, rarely going beyond $2 \%$. Further, the bias does not seem to be affected by the extent of dependence. The FE of the scale parameter MLEs are close to $100 \%$ regardless of the values of $m$ and $\eta$, demonstrating close agreement between asymptotic and finite-sample variability. It seems, however, that the large-sample variance of $\hat{\beta}_{1}, \hat{\beta}_{2}$ severely underestimate the true variability for small to moderate $m$. The tables also clearly demonstrate that ignoring the dependence results in severe underestimation of the variability of the MLEs of the scale parameters, even for small to moderate $\eta$. Indeed, as exhibited in Theorem 1, the amount of underestimation increases with the magnitudes of $\eta$ as well as the scale parameters. 

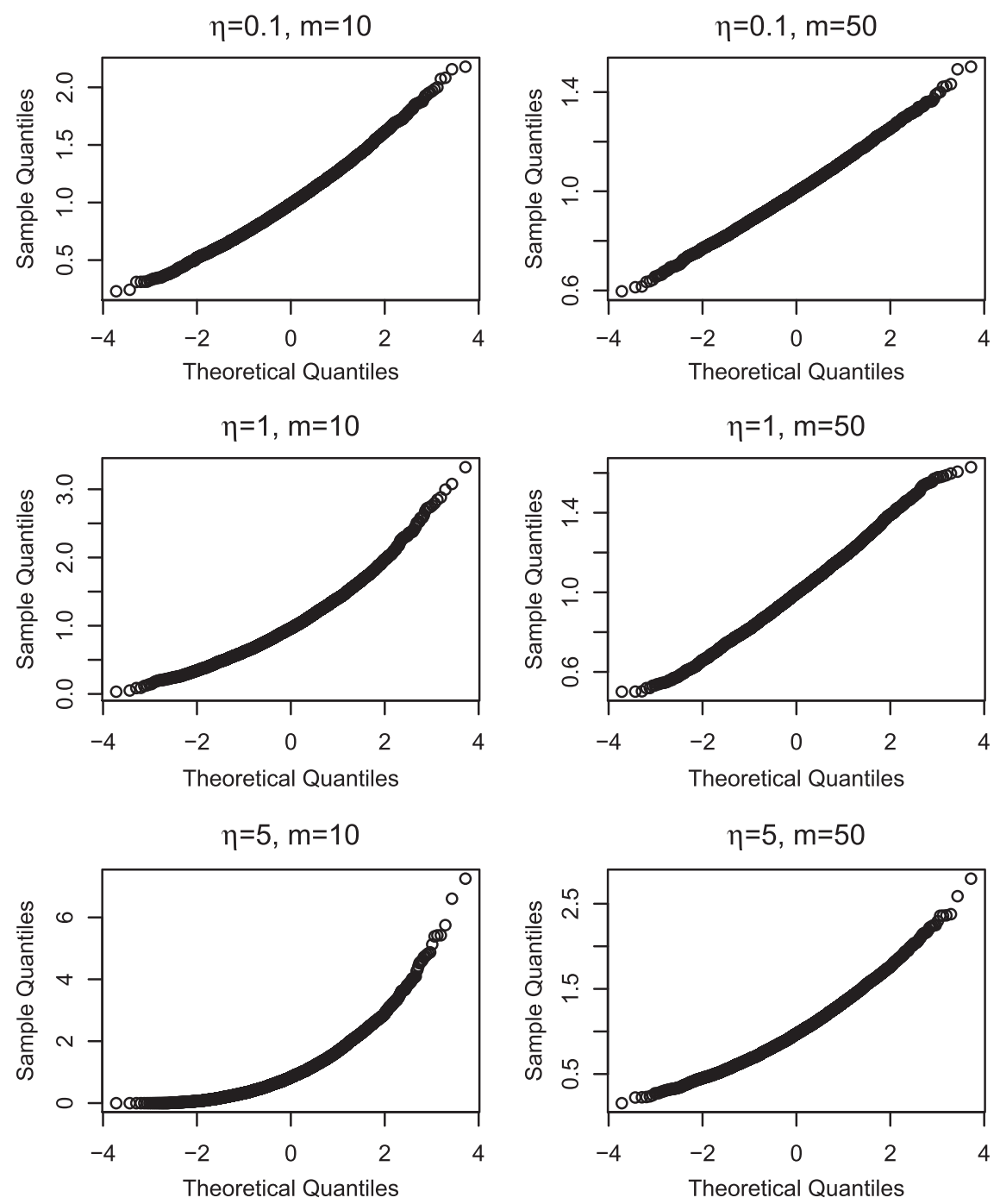

Figure 1. Q-Q plots of $\hat{\mu}_{1}$ for varying $m$ and $\eta$.

Figure 1 and Figure 2 show the Q-Q plots for $\left(\hat{\mu}_{1}, \hat{\beta}_{1}\right)$ for different sets of $m$ and $\eta$ and with the true parameter vector being $\mu_{1}=1, \beta_{1}=0.75, \mu_{2}=0.3, \beta_{2}=1.25$. The findings for $\left(\hat{\mu}_{2}, \hat{\beta}_{2}\right)$ are similar. As $\eta$ increases, the deviation from normality for both $\hat{\mu}_{1}$ and $\hat{\beta}_{1}$ become increasingly prominent when data from only a small number of systems are available. For the distribution of $\hat{\beta}_{1}$, however, the conformity to normality is realized at a faster rate than that for $\hat{\mu}_{1}$.

\section{Automobile warranty data analysis}

The motivating example for the model and methodology discussed in this article is a dataset comprising of recurrent failure history of a fleet of automobiles. The outcome of interest is repeated mileages at failure for multiple vehicles of a certain model and make, obtained from a warranty claim database, that also documents the labor code associated with the failure. In order to verify the conformity to a PLP, logarithm of cumulative number of failures is plotted against the cumulative mileage at failure for the overall failure history pooled across the individual prototypes. For three broad groupings of the labor codes, these plots, known in the literature as Duane plots [14] show a fairly linear pattern (Figure 3), confirming the plausibility of the parametric model used in this article.

The data, shown in Table IV, are real, but the source and specifics are masked for de-identification purposes. The database consists of recurrent failure history of 456 vehicles. We consider a single Type-I censoring at 3000 miles. Fourteen different labor codes of the warranty claims of each vehicle are recorded with mileage at filing. Ideally, the grouping should be dictated by engineering judgement and a knowledge of the component interactions. This was not possible here due to the 

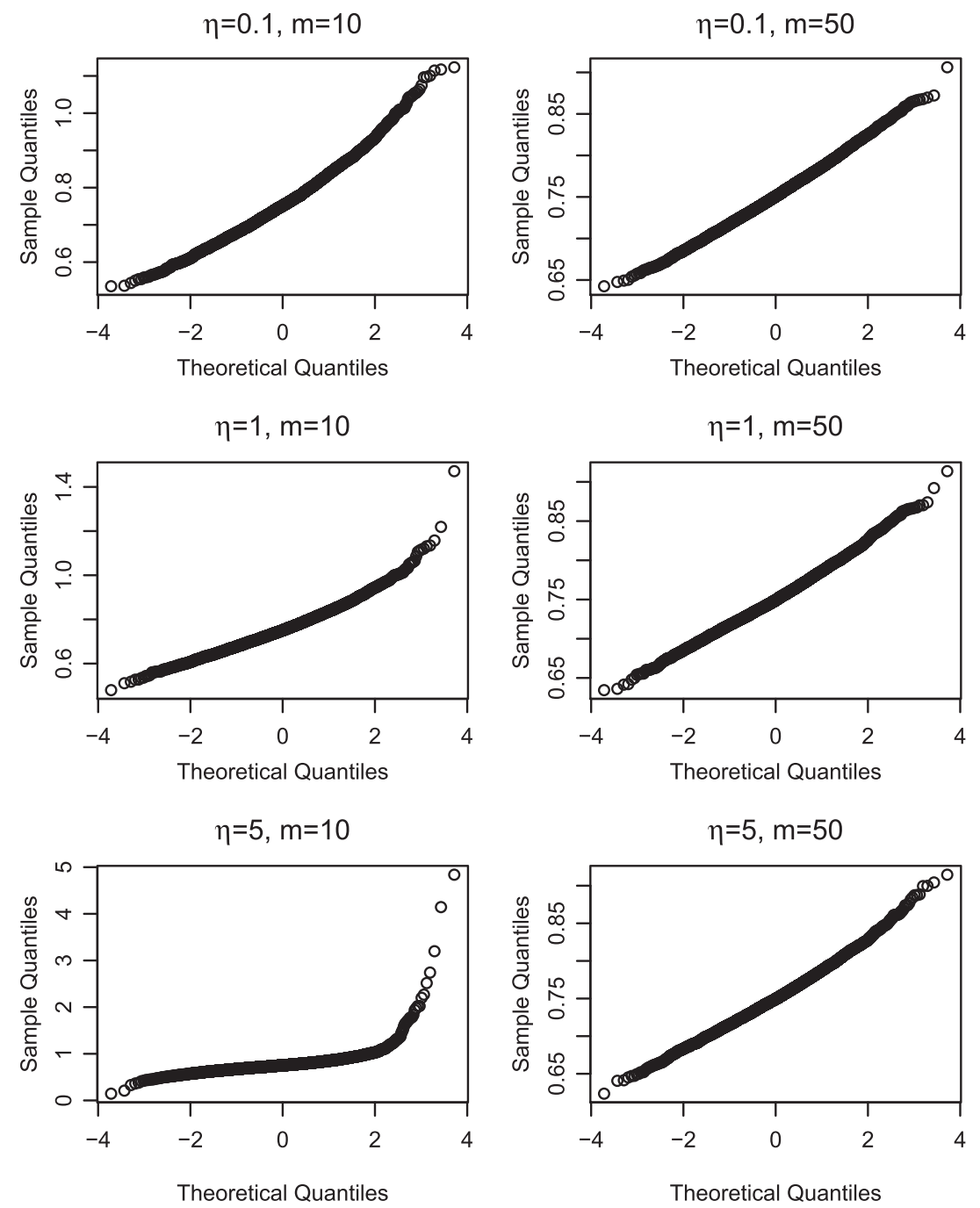

Figure 2. Q-Q plots of $\hat{\beta}_{1}$ for varying $m$ and $\eta$.
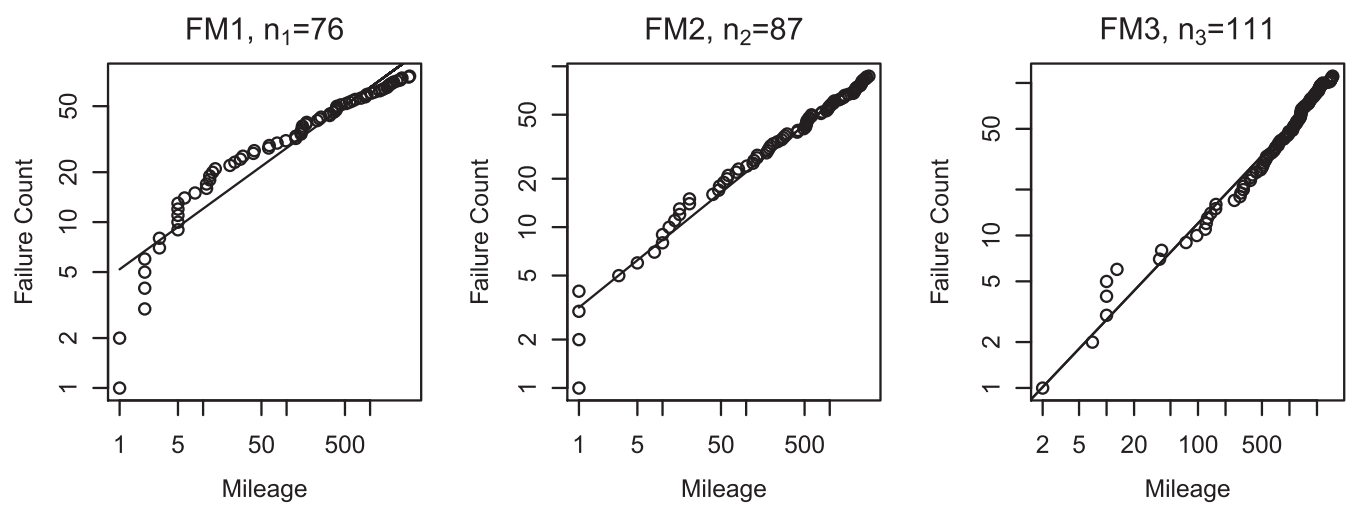

Figure 3. Plots in log-log scale of mileage versus failure count for the automobile warranty data; also indicated are the total number of failures corresponding to the failure mode (FM). 


\begin{tabular}{|c|c|c|c|c|c|c|c|c|c|c|c|c|c|c|c|c|c|c|c|c|}
\hline No. & Mil. & FM & No. & Mil. & FM & No. & Mil. & FM & No. & Mil. & FM & No. & Mil. & FM & No. & Mil. & FM & No. & Mil. & FM \\
\hline 1 & 12 & 1 & 26 & 777 & 1 & 51 & 2205 & 3 & 80 & 3 & 1 & 108 & 174 & 1 & 131 & 1 & 1 & 161 & 157 & 3 \\
\hline 1 & 514 & 2 & 26 & 985 & 3 & 52 & 294 & 3 & 81 & 10 & 3 & 108 & 2850 & 3 & 132 & 2270 & 3 & 161 & 515 & 3 \\
\hline 1 & 2411 & 1 & 26 & 1107 & 2 & 53 & 1488 & 1 & 82 & 40 & 1 & 109 & 1140 & 3 & 133 & 1250 & 3 & 161 & 1380 & 3 \\
\hline 2 & 484 & 3 & 26 & 1303 & 1 & 54 & 54 & 2 & 83 & 127 & 3 & 109 & 2720 & 2 & 133 & 1842 & 1 & 161 & 1861 & 3 \\
\hline 2 & 525 & 3 & 26 & 1499 & 2 & 55 & 5 & 1 & 84 & 1200 & 3 & 110 & 129 & 1 & 134 & 1081 & 3 & 161 & 1953 & 2 \\
\hline 3 & 2800 & 3 & 26 & 2420 & 2 & 56 & 519 & 2 & 85 & 135 & 2 & 110 & 156 & 3 & 134 & 1457 & 2 & 161 & 2296 & 1 \\
\hline 4 & 1347 & 3 & 26 & 2446 & 2 & 57 & 61 & 2 & 85 & 532 & 2 & 111 & 367 & 1 & 135 & 196 & 2 & 161 & 2313 & 3 \\
\hline 5 & 14 & 1 & 27 & 2105 & 2 & 57 & 293 & 2 & 86 & 38 & 3 & 112 & 5 & 2 & 136 & 1 & 2 & 161 & 2964 & 3 \\
\hline 5 & 558 & 2 & 28 & 1535 & 3 & 57 & 1355 & 3 & 86 & 1137 & 2 & 113 & 40 & 3 & 137 & 13 & 3 & 162 & 1037 & 1 \\
\hline 5 & 559 & 3 & 29 & 98 & 1 & 57 & 1911 & 1 & 86 & 1668 & 2 & 114 & 3 & 1 & 138 & 696 & 3 & 163 & 59 & 2 \\
\hline 5 & 1599 & 1 & 29 & 120 & 3 & 58 & 503 & 3 & 87 & 233 & 1 & 115 & 81 & 2 & 138 & 2972 & 1 & 164 & 2104 & 3 \\
\hline 6 & 10 & 3 & 30 & 2028 & 2 & 59 & 121 & 2 & 88 & 1181 & 3 & 115 & 97 & 3 & 139 & 2 & 1 & 165 & 374 & 3 \\
\hline 7 & 28 & 1 & 30 & 2329 & 2 & 60 & 21 & 2 & 88 & 2840 & 3 & 116 & 2 & 1 & 140 & 5 & 1 & 165 & 408 & 2 \\
\hline 8 & 1 & 1 & 31 & 1507 & 2 & 61 & 1135 & 1 & 89 & 2898 & 1 & 117 & 1200 & 3 & 141 & 2574 & 2 & 165 & 436 & 3 \\
\hline 9 & 102 & 2 & 32 & 1370 & 2 & 62 & 16 & 2 & 90 & 123 & 3 & 118 & 1662 & 3 & 142 & 7 & 3 & 165 & 490 & 2 \\
\hline 10 & 1812 & 3 & 33 & 2164 & 3 & 63 & 1603 & 3 & 90 & 138 & 3 & 119 & 1733 & 1 & 142 & 816 & 2 & 165 & 1287 & 2 \\
\hline 10 & 1938 & 3 & 34 & 914 & 2 & 64 & 801 & 3 & 90 & 2966 & 3 & 120 & 887 & 1 & 143 & 41 & 1 & 165 & 1631 & 3 \\
\hline 11 & 12 & 1 & 35 & 10 & 3 & 65 & 155 & 1 & 91 & 189 & 2 & 120 & 938 & 3 & 143 & 2750 & 3 & 165 & 2387 & 2 \\
\hline 12 & 569 & 1 & 35 & 1622 & 1 & 66 & 76 & 2 & 92 & 10 & 2 & 121 & 288 & 3 & 144 & 535 & 2 & 165 & 2801 & 3 \\
\hline 13 & 2097 & 3 & 36 & 525 & 2 & 66 & 77 & 1 & 93 & 870 & 3 & 121 & 2083 & 3 & 144 & 1322 & 3 & 166 & 5 & 1 \\
\hline 14 & 776 & 3 & 37 & 154 & 1 & 66 & 153 & 1 & 94 & 1 & 2 & 122 & 325 & 3 & 144 & 1692 & 3 & 166 & 543 & 3 \\
\hline 14 & 979 & 2 & 37 & 1979 & 3 & 67 & 149 & 1 & 94 & 13 & 1 & 122 & 400 & 1 & 145 & 10 & 2 & 167 & 237 & 2 \\
\hline 15 & 309 & 2 & 38 & 149 & 1 & 67 & 2127 & 3 & 95 & 620 & 1 & 123 & 1961 & 2 & 146 & 562 & 3 & 167 & 2424 & 2 \\
\hline 15 & 2719 & 2 & 38 & 210 & 2 & 68 & 265 & 2 & 96 & 1086 & 3 & 124 & 170 & 1 & 146 & 665 & 1 & 168 & 416 & 2 \\
\hline 16 & 1015 & 2 & 39 & 1097 & 3 & 69 & 1912 & 2 & 97 & 1722 & 3 & 125 & 1116 & 3 & 146 & 2868 & 3 & 169 & 396 & 1 \\
\hline 16 & 1440 & 3 & 40 & 3 & 2 & 70 & 24 & 1 & 98 & 40 & 2 & 126 & 520 & 1 & 147 & 136 & 2 & 169 & 1315 & 3 \\
\hline 16 & 2055 & 2 & 40 & 12 & 2 & 71 & 5 & 1 & 98 & 1041 & 2 & 126 & 709 & 3 & 148 & 2883 & 3 & 169 & 1350 & 3 \\
\hline 17 & 14 & 2 & 41 & 2176 & 3 & 72 & 1301 & 3 & 98 & 1393 & 1 & 126 & 878 & 1 & 149 & 259 & 1 & 169 & 1693 & 3 \\
\hline 17 & 16 & 2 & 42 & 604 & 3 & 72 & 1333 & 3 & 98 & 1447 & 3 & 126 & 2091 & 3 & 150 & 920 & 1 & 169 & 1902 & 3 \\
\hline 17 & 62 & 1 & 43 & 250 & 3 & 73 & 2 & 1 & 99 & 331 & 1 & 126 & 2316 & 3 & 151 & 975 & 3 & 169 & 2016 & 2 \\
\hline 17 & 74 & 3 & 44 & 2198 & 2 & 73 & 1779 & 3 & 100 & 313 & 3 & 127 & 1270 & 3 & 152 & 11 & 1 & 170 & 279 & 2 \\
\hline 17 & 1696 & 1 & 45 & 8 & 1 & 74 & 6 & 1 & 100 & 329 & 1 & 128 & 1104 & 2 & 153 & 48 & 2 & 170 & 1330 & 3 \\
\hline 18 & 2144 & 3 & 45 & 397 & 1 & 74 & 984 & 3 & 101 & 756 & 3 & 129 & 21 & 2 & 154 & 2338 & 1 & 171 & 677 & 3 \\
\hline 19 & 787 & 2 & 46 & 604 & 2 & 74 & 1854 & 3 & 102 & 447 & 1 & 129 & 61 & 1 & 155 & 1 & 2 & 172 & 1633 & 3 \\
\hline 20 & 30 & 1 & 47 & 248 & 1 & 75 & 21 & 1 & 103 & 2 & 1 & 129 & 175 & 2 & 156 & 2324 & 2 & & & \\
\hline 21 & 960 & 2 & 47 & 2939 & 2 & 76 & 2 & 3 & 104 & 1343 & 3 & 129 & 182 & 2 & 157 & 1957 & 3 & & & \\
\hline 22 & 2524 & 2 & 48 & 129 & 1 & 77 & 753 & 3 & 105 & 11 & 1 & 129 & 378 & 3 & 158 & 2606 & 3 & & & \\
\hline 23 & 5 & 1 & 48 & 567 & 2 & 77 & 944 & 2 & 106 & 313 & 3 & 129 & 386 & 1 & 159 & 888 & 3 & & & \\
\hline 24 & 2090 & 1 & 49 & 8 & 2 & 78 & 124 & 2 & 106 & 645 & 3 & 129 & 1512 & 3 & 160 & 2040 & 3 & & & \\
\hline 25 & 1 & 2 & 50 & 395 & 3 & 79 & 2921 & 3 & 107 & 2767 & 2 & 130 & 604 & 2 & 161 & 47 & 2 & & & \\
\hline
\end{tabular}

No., vehicle number; Mil., mileage; FM, failure mode (1-3).

absence of a specific description of the component associated with a labor code. We thus determined the grouping on the basis of rate of failures. For all 14 individual labor codes, separate PLP were fit. Subsequently, the labor codes were combined into three broad groups of failure modes FM1-FM3, where FM1 comprises of labor codes with shape parameters ranging between 0.2 and 0.36 , FM2 covers labor codes with shape parameter estimates between 0.4 and 0.55 , whereas FM3 combines the remaining codes that have the slowest rate of growth with shape parameter estimates varying between 0.7 and 0.93 . Less than $4 \%$ of the vehicles had event times with ties across different failure modes. Although this is not a deterrent to the analysis, we dropped these vehicles from consideration in order to make a cleaner presentation. Table IV presents the data that have at least one documented record of warranty repair. In addition, there are 267 vehicles that have no claim during the observation period, yielding a total of 439 vehicles that were considered for analysis. Pursuant to the failure mode grouping, we observe 76, 87, and 111 failures due to FM1, FM2, and FM3, respectively.

Under the assumption of PLP governing the failure processes corresponding to the modes, the MLEs of the model parameters are estimated as 


$$
\hat{\mu}_{1}=1.519 \times 10^{-2}, \hat{\beta}_{1}=0.304, \hat{\mu}_{2}=7.215 \times 10^{-3}, \hat{\beta}_{2}=0.414, \hat{\mu}_{3}=8.974 \times 10^{-4}, \hat{\beta}_{3}=0.705
$$

We assume a dependence structure induced by a gamma distributed frailty random variable with mean 1 and variance $\eta$. Applying Theorem 1, the large-sample 95\% confidence interval for $\beta_{1}, \beta_{2}$, and $\beta_{3}$ are given by $(0.236,0.372),(0.327$, $0.501)$, and $(0.573,0.836)$, respectively, indicating reliability growth in each mode, consistent with the plots in Figure 1. Using the asymptotic independence of $\hat{\beta}_{1}$ and $\hat{\beta}_{2}$, a large-sample $95 \%$ confidence interval for $\beta_{1}-\beta_{2}$ is given by

$$
\left(\hat{\beta}_{1}-\hat{\beta}_{2}\right) \pm 1.96 \sqrt{\widehat{\operatorname{AVar}}\left(\hat{\beta}_{1}\right)+\widehat{\operatorname{AVar}}\left(\hat{\beta}_{2}\right)}=(-0.220,0.001)
$$

where $\widehat{\mathrm{AVar}}$ indicates estimated asymptotic variance. Similarly, the asymptotic $95 \%$ confidence intervals for $\beta_{1}-\beta_{3}$ and $\beta_{2}-\beta_{3}$ are obtained as $(-0.548,-0.253)$ and $(-0.448,-0.133)$, respectively. The dependence parameter (frailty variance) $\eta$ affects the distribution of the scale parameter $\mu$. It is estimated for our data as $\hat{\eta}=5.450$. Using the entries of the large-sample variance-covariance matrix of $\hat{\mu}_{1}, \hat{\mu}_{2}$, and $\hat{\mu}_{3}$ from Theorem 1, the $95 \%$ asymptotic confidence intervals for $\mu_{1}-\mu_{2}, \mu_{1}-\mu_{3}$, and $\mu_{2}-\mu_{3}$ are $(-0.003,0.019),(0.003,0.025)$, and $(-0.001,0.014)$, respectively (Table V).

We carried out a parametric bootstrap procedure to assess the closeness of the large-sample results to a seemingly more honest description of the random behavior of the MLEs. In order to generate the $b^{\text {th }}$ bootstrap estimates of $\mu_{1}, \mu_{2}, \mu_{3}, \beta_{1}, \beta_{2}, \beta_{3}$, we carry out steps identical to those in Steps 1-5 in Section 5, with $\mu_{j}, \beta_{j}$ replaced by $\hat{\mu}_{j}, \hat{\beta}_{j}, j=1,2,3$.

All subsequent calculations are based on 10000 bootstrap samples. The associated $95 \%$ confidence intervals using

\begin{tabular}{|c|c|c|}
\hline \multirow{3}{*}{ Parameter } & \multicolumn{2}{|c|}{ 95\% Confidence interval } \\
\hline & & \\
\hline & Asymptotic & Bootstrap \\
\hline$\mu_{1}$ & $(0.6,2.5) \times 10^{-2}$ & $(0.9,2.3) \times 10^{-2}$ \\
\hline$\beta_{1}$ & $(0.236,0.372)$ & $(0.248,0.375)$ \\
\hline$\mu_{2}$ & $(0.2,1.3) \times 10^{-2}$ & $(0.3,1.2) \times 10^{-2}$ \\
\hline$\beta_{2}$ & $(0.327,0.50)$ & $(0.337,0.511)$ \\
\hline$\mu_{3}$ & $(0.0,1.9) \times 10^{-3}$ & $(0.2,2.2) \times 10^{-3}$ \\
\hline$\beta_{3}$ & $(0.573,0.836)$ & $(0.582,0.845)$ \\
\hline$\mu_{1}-\mu_{2}$ & $(-0.003,0.019)$ & $(0.000,0.016)$ \\
\hline$\mu_{1}-\mu_{3}$ & $(0.003,0.025)$ & $(0.008,0.022)$ \\
\hline$\mu_{2}-\mu_{3}$ & $(-0.001,0.014)$ & $(0.002,0.011)$ \\
\hline$\beta_{1}-\beta_{2}$ & $(-0.220,0.001)$ & $(-0.223,-0.005)$ \\
\hline$\beta_{1}-\beta_{3}$ & $(-0.548,-0.253)$ & $(-0.553,-0.259)$ \\
\hline$\beta_{2}-\beta_{3}$ & $(-0.448,-0.133)$ & $(-0.453,-0.137)$ \\
\hline
\end{tabular}
the bias-corrected percentile bootstrap method ([21], Eq. (7.9)) are presented in Table V along with the ones based on

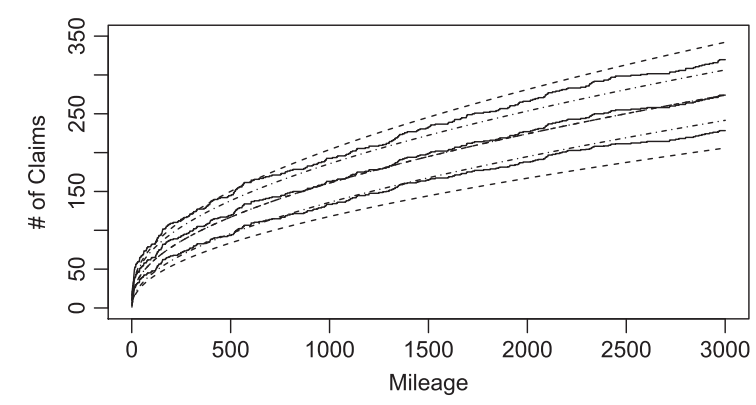

Figure 4. The 95\% large-sample confidence bands for estimated number of claims in the warranty data based on nonparametric (solid), power law process (PLP) with frailty (dashed), and PLP without frailty (dash-with-dots). 
large-sample theory. Generally, the bootstrap-based intervals are in close agreement with the large-sample version, which is not surprising due to the symmetric nature of the estimated distributions of the MLEs (picture not shown).

Figure 4 shows $95 \%$ pointwise asymptotic confidence intervals for numbers of claims from all 439 vehicles based on (11) as well as the nonparametric analog in (14). They exhibit quite similar patterns, although the nonparametric confidence band is slightly narrower than its parametric counterpart owing to the fact that the nonparametric one does not explicitly account for the variability in the frailty variable. For comparison purposes, we have also included the band based on a PLP model without frailty (dash-with-dots), which is the narrowest, as expected.

\section{Concluding remarks}

In this article, we have provided an investigation of statistical inference for failure data arising from multiple repairable systems that are subject to competing risks. The setup is pertinent to a failure mode effect analysis program often pursued for complex multicomponent systems. The main focus of the article is on carrying out the inference under a shared frailty structure that induces dependence across the component processes. Shared frailty model is essentially a random effects model with two different sources of variation [16]. The variability of $Z_{i}$ in (2) represents the between-systems variation, whereas the variability reflected through the intensity function $\mu(t)$ represents variation within the repeat observations of the $i$-th system. Shared random effects that the frailty formulation is akin to are also quite commonly employed in joint modeling of repeated measures and time to event data [22]. Of course, shared frailty is not the only way to induce dependence among components. One can perhaps directly appeal to the counting processes and model them in a multivariate way. That would certainly be the preferred way to carry out inference if an assessment of the degree of dependence among the components were sought. Here, our main interest lies on the inference of the model parameters. Treating the association between the components as a nuisance parameter via frailty thus seems to be a natural approach in this case.

The discourse in this article has been confined to PLP with a gamma frailty that can be construed as an extension to a popular parametric framework in this context. The result in Theorem 1 is presented for the single censoring case. The extension to the multiple censoring case with differing $\tau_{i}$ is substantially more cumbersome. In particular, the MLEs no longer possess the attractive closed-form structures. In this case, one has to take recourse to iterative algorithms such as NewtonRaphson to obtain the MLEs. As long as the mean model is specified correctly for the component recurrent processes, the general theory of estimating equations is conceivably applicable to ensure the large-sample normality of the solutions to the likelihood equations. For a general parametric counting process formulation of a recurrent event framework with differing censoring times, Lawless and Nadeau [19] develop the large-sample theory of the MLEs in the non-competing risks case. The generalization to the competing risks case should follow along similar steps. The insights into the behavior of the MLEs for the specific parametric formulation of PLP, however, remain unexplored.

Our simulation findings indicate that a moderate to large frailty variance coupled with the availability of a small number of system prototypes have a significant impact on the standard errors of the ML estimators. In view of this, we recommend estimating the dependence parameter $\eta$ first when analyzing a dataset under the frailty induced PLP framework. If the estimate $\hat{\eta}$ exceeds 1 , or the number of systems tested is small (say $m \leqslant 30$ ), we recommend using the bootstrap met for inference along the lines described in Sections 5-6.

Some earlier investigations have considered PLP in the context of complex multicomponent systems that is akin to a competing risks framework. Pievatolo and Ruggeri [23] advanced a Bayesian approach in such a framework where the repair history of the individual components conform to PLPs. A recent article by Hong et al. [24] investigated unavailability of a system with repair pattern that can be viewed as a superposition of PLPs. One important feature of these work is their formulation of component failure processes as independent. The premise we investigate in this paper assumes a shared frailty structure that induces a dependence among the component processes.

We strongly believe that the investigation presented herein provides an important extension to a widely used parametric model for repairable system failure. In the area of software reliability, parametric formulations of intensity function different from PLP or classes of parametric models of gap times are often pursued. The general approach pursued in this article can be adapted to these framework as well. As indicated earlier, methodologies exist for carrying out inference in a nonparametric setting in the context of analyzing recurrent events under competing risks. The inference in the nonparametric case is restricted to mean or rate of cause-specific recurrence and is not valid for time beyond the largest censoring time. The inference in a parametric setting, on the other hand, is more comprehensive and can be extrapolated beyond the observation point. The trade-off, of course, lies in the robustness of model assumptions. In recurrent data from human trials where it is desired to model the effect of prognostic risk factors on the mean or rate, a semi-parametric regression model of the mean function may be a preferred alternative to a fully parametric setting. Such models can be conceived as extensions of similar models and methodologies pursued in the nonrecurrent framework [25]. 


\section{Appendix}

\section{Proof of Theorem 1}

Define $S_{l m}=\beta_{l} \sum_{i=1}^{m} \sum_{j=1}^{n_{i}} I\left(\delta_{i j}=l\right) \log \left(\tau / t_{i j}\right)$ for $l=k, k^{\prime}$. Define by $n_{\cdot l}=\sum_{i=1}^{m} n_{i l}$ the observed number of $l$-mode failures. Given $n_{\cdot l}$ and $z_{i}$ 's, we have $S_{l m} \sim \operatorname{Gamma}\left(n_{\cdot l}, 1\right)$ with $S_{k m}$ and $S_{k^{\prime} m}$ mutually independent. Using strong law of large numbers, it follows that $n_{\cdot l} / m \mu_{l} \tau^{\beta_{l}} \stackrel{a . s .}{\rightarrow} 1$ as $m \rightarrow \infty_{d}$. Hence, $n_{\cdot l} \stackrel{a . s .}{\rightarrow} \infty$ as $m \rightarrow \infty$. Furthermore, if we let $v_{l m}=\left(S_{l m}-n_{\cdot l}\right) / \sqrt{n_{\cdot l}}$, then using central limit theorem $\left(v_{k m}, v_{k^{\prime} m}\right)^{\prime} \stackrel{d}{\rightarrow} N_{2}\left(\mathbf{0}, \mathbf{I}_{2}\right)$ as $m \rightarrow \infty$. Because the conditional distribution does not depend on $n_{\cdot k}, n_{\cdot k^{\prime}}$, it is also the unconditional distribution. Noting that $S_{l m}=n_{\cdot l} \beta_{l} / \hat{\beta}_{l}, v_{l m}$ can be re-expressed as $v_{l m}=\sqrt{n_{\cdot l}}\left(\beta_{l}-\hat{\beta}_{l}\right) / \hat{\beta}_{l}$. Consequently,

$$
\sqrt{m}\left(\hat{\beta}_{l}-\beta_{l}\right)=\frac{-\hat{\beta}_{l}}{\sqrt{n_{\cdot l} / m}} v_{l m}, \quad l=k, k^{\prime}
$$

Using the large-sample distribution of $\left(v_{k m}, v_{k^{\prime} m}\right)^{\prime}$ along with the facts that $\hat{\beta}_{l} \stackrel{p}{\rightarrow} \beta_{l}$ and $n_{\cdot l} / m \stackrel{p}{\rightarrow} \mu_{l} \tau^{\beta_{l}}$, we have

$$
\left(\hat{\beta}_{k}-\beta_{k}, \hat{\beta}_{k^{\prime}}-\beta_{k^{\prime}}\right)^{\prime} \stackrel{d}{\rightarrow} N_{2}\left(\mathbf{0},\left[\begin{array}{cc}
\beta_{k}^{2} /\left(\mu_{k} \tau^{\beta_{k}}\right) & 0 \\
0 & \beta_{k^{\prime}}^{2} /\left(\mu_{k^{\prime}} \tau^{\beta_{k^{\prime}}}\right)
\end{array}\right]\right)
$$

Define further $u_{l m}=\sqrt{m} \log \left(n_{\cdot l} / m \mu_{l} \tau^{\beta_{l}}\right)$ for $l=k, k^{\prime}$ and $u_{m}=\left(u_{k m}, u_{k^{\prime} m}\right)^{\prime}$. Then, by using the bivariate central limit theorem, we have

$$
u_{m} \stackrel{d}{\rightarrow} N\left(\mathbf{0},\left[\begin{array}{cc}
\frac{1+\eta \mu_{k} \tau^{\beta_{k}}}{\mu_{k}} & \eta \\
\eta & \frac{1+\eta \mu_{k}^{\prime} \tau^{\prime} \tau_{k}^{\prime}}{\mu_{k}^{\prime}}
\end{array}\right]\right), \text { as } m \rightarrow \infty,
$$

where we used the expressions from (4) and (5). Rewriting $\hat{\mu}_{l}$ with some intended manipulation, we see

$$
\log \hat{\mu}_{l}=\log \mu_{l}+\frac{u_{l m}}{\sqrt{m}}+\left(\beta_{l}-\hat{\beta}_{l}\right) \log \tau
$$

which upon using (A.1) and a Taylor Series expansion yield

$$
\sqrt{m}\left(\hat{\mu}_{l}-\mu_{l}\right)=\frac{\mu_{l} \beta_{l} \log \tau}{\sqrt{\mu_{l} \tau^{\beta_{l}}}} v_{l m}+\mu_{l} u_{l m}+o_{p}(1), l=k, k^{\prime}
$$

The result follows from (A.1) and (A.2), upon noting that $v_{l m}$ and $u_{l m}$ are asymptotically independent.

\section{Acknowledgements}

The authors gratefully acknowledge the advise of the review team that helped immensely in preparing a high-quality manuscript. The authors further acknowledge the guidance of Professor Vijay Nair at the University of Michigan during the development phase of the project.

\section{References}

1. Crowder M. Classical Competing Risks. Chapman \& Hall/CRC: New York, 2001.

2. Pintilie M. Competing Risks: A Practical Perspective (Statistics in Practice). John Wiley \& Sons Ltd: West Sussex, England, 2006.

3. Majumdar SK. An optimum maintenance strategy for a vertical boring machine system. Operational Research Society of India 1993; 30:344-365. 
4. Langseth H, Lindqvist BH. Competing risks for repairable systems: a data study. Journal of Statistical Planning and Inference 2006; 136: $1687-1700$.

5. Lawless JF, Wigg MB, Tuli S, Drake J, Lamberti-Pasculli M. Analysis of repeated failures or durations, with application to shunt failures for patients with paediatric hydrocephalus. Applied Statistics 2001; 50:449-465.

6. Dauxois J-Y, Sencey S. Non-parametric tests for recurrent events under competing risks. Scandinavian Journal of Statistics 2009; 36:649-670.

7. Bain LJ. Statistical Analysis of Reliability and Life-Testing Models: Theory and Methods (2nd edn). Marcel Dekker: New York, 1978.

8. Rigdon SE, Basu AP. Statistical Methods for the Reliability of Repairable Systems. Wiley: New York, 2000.

9. Crow LH. Reliability analysis for complex, repairable systems. In Reliability and Biometry, Proschan F, Serfling RJ (eds). SIAM: Philadelphia, $1974 ; 379-410$.

10. Somboonsavatdee A, Sen A. Statistical inference for power law process with competing risks. Technometrics 2014. http://amstat.tandfonline. com/action/showCitFormats?doi=10.1080/00401706.2014.902772.

11. Elandt-Johnson R. Conditional failure time distributions under competing risk theory with dependent failure times and proportional hazard rates. Scandinavian Actuarial Journal 1976; 59:37-51.

12. Taylor L, Peña EA. Parametric estimation in a recurrent competing risks model. Journal of Iranian Statistical Society 2013; 12:153-181.

13. Peña EA, Strawderman RL, Hollander M. Nonparametric estimation with recurrent event data. Journal of the American Statistical Association 2001; 96:1299-1315.

14. Duane JT. Learning curve approach to reliability monitoring. IEEE Transactions on Aerospace 1964; 2:563-566.

15. Vaupel JW, Manton KG, Stallard E. The impact of heterogeneity in individual frailty on the dynamics of mortality. Demographics 1979; 16: 439-454.

16. Hougaard P. Analysis of Multivariate Survival Data. Springer: New York, 2000.

17. Elbers C, Ridder G. True and spurious duration dependence: the identifiability of the proportional hazard model. The Review of Economic Studies 1982; 49:403-409.

18. Ghosh D, Lin DY. Nonparametric analysis of recurrent events and death. Biometrics 2000; 56:554-562.

19. Lawless JF, Nadeau C. Some simple robust methods for the analysis of recurrent events. Technometrics 1995; 37:158-168.

20. Lin DY, Wei LJ, Yang I, Ying Z. Semiparametric regression for the mean and rate functions of recurrent events. Journal of the Royal Statistical Society. Series B 2000; 62:771-730.

21. Efron B, Tibshirani R. Bootstrap methods for standard errors, confidence intervals, and other measures of statistical accuracy. Statistical Science 1986; 1:54-75.

22. McCrink LM, Marshal AH, Cairns KJ. Advances in joint modelling: A review of recent developments with application to the survival of end stage renal disease patients. International Statistical Review 2013; 81:249-269.

23. Pievatolo A, Ruggeri F. Bayesian reliability analysis of complex repairable systems. Applied Stochastic Models in Business and Industry 2004; 20:253-264.

24. Hong Y, Li M, Osborn B. System unavailability analysis based on window-observed recurrent event data. Applied Stochastic Models in Business and Industry 2013. DOI: 10.1002/asmb.1984.

25. Gorfine M, Hsu L. Frailty-based competing risks model for multivariate survival data. Biometrics 2011; 67:415-426. 